\title{
Near-Infrared Light Stimuli-responsive Synergistic Therapy Nanoplatforms Based on the Coordination of Tellurium-containing Block Polymer and Cisplatin for Cancer Treatment
}

Feng $\mathrm{Li}^{\mathrm{a}}$, Tianyu $\mathrm{Li}^{\mathrm{a}}$, Wei Cao ${ }^{\mathrm{a}}$, Lu Wang ${ }^{\mathrm{a}}$, and Huaping $\mathrm{Xu}^{\mathrm{a}}{ }{ }^{*}$

${ }^{\text {a }}$ Key Lab of Organic Optoelectronics \& Molecular Engineering, Department of Chemistry, Tsinghua University, Beijing 100084, China.

E-mail: xuhuaping@mail.tsinghua.edu.cn

Keywords: tellurium-containing polymer, cisplatin, coordination chemistry, drugdelivery, photo-responsive, cancer treatment

Abstract: Cisplatin (CDDP) has received worldwide approval for clinical use in the past decades. However, its development in cancer chemotherapy was overshadowed by severe side effects and drug resistance. Herein, we developed a CDDP drug delivery system with high encapsulation efficiency and near-infrared light stimuliresponsive drug release properties based on the coordination of novel telluriumcontaining block polymer (PEG-PUTe-PEG) and CDDP. The nanocarriers made from PEG-PUTe-PEG were loaded with CDDP and indocyanine green (ICG) simultaneously. The coordination chemistry between CDDP and tellurium guaranteed the nanocarrier a high stability in plasma and prolonged circulation time in vivo by reducing possible penetration of water molecule into the nanoparticles. Under the stimuli of a near-infrared laser, a amount of ROS can be generated by irradiation of ICG. The tellurium is easily oxidized by ROS because of the low electronegativity of tellurium. The CDDP could be rapidly released from the nanocarriers along with the 
oxidation of the tellurium at the tumor sites as the oxidized tellurium will weaken the coordination interaction with CDDP. In addition, the encapsulated ICG played a synergistic antitumor effect through photothermal effect with mild laser irradiation. The integrated strategy achieved higher antitumor efficacy and showed minimal side effects compared with the CDDP alone.

\section{Introduction}

Cisplatin (CDDP), a leading platinum-based anticancer drug, has been widely used for the treatment of various solid tumors since it was approved by FDA in 1978 for cancer treatment.[1, 2] Although platinum (Pt)-based anticancer drugs have gained great success in cancer treatment, there are still concerns associated with the use of platinum-based anticancer drugs, on account of their significant side effects and drug resistance, which may markedly limit their clinical applications.[3-6]

Encapsulation of drugs in nanocarriers that target tumor tissues promises to be an effective method to mitigate the side effects of conventional chemotherapy.[7-15] The delivery of CDDP via polymers or liposome has been extensively exploited.[15-19] Physical encapsulation of drugs into a polymeric or liposome carrier is a common strategy for CDDP.[15-17] However, the major drawback of physical encapsulation is the low encapsulation efficiency, which is usually limited by low hydrophilicity and lipophilicity properties. Besides, the unstable nanomedicine formulations can leak CDDP rapidly during storage or within the plasma in vivo, often with an initial burst release. In contrast to physical encapsulation, chemical conjugation of the platinum- 
based drugs to the polymer leads to better stability during the circulation period and to avoid the leakage before reaching the tumors.[20-23] Unfortunately, the highly stable drug-polymer conjugates usually have uncontrolled drug release property and are even consequently inactive against tumor cells.[24] Stimuli-responsive chemical bonds between drugs and the polymeric backbone have been utilized for controlled release of drugs.[25-29] However, the field of platinum-based drugs is still attractive to researchers who try to explore more effective delivery approaches.

Tellurium-containing polymers have been employed to construct nanoparticles with ultra-sensitive ROS responsiveness due to the low electronegativity of tellurium and have also demonstrated great potentials as innovative promising biomaterials. [30-32] The coordination chemistry between platinum and tellurium opens new opportunities for platinum-based drugs delivery and endows the tellurium-containing polymers with great potential in platinum drugs delivery and controlled drug release in vivo. [33-36] Indocyanine green (ICG), a near-infrared tricarbocyanine dye, has obtained approval from the US Food and Drug Administration (FDA) as a clinical diagnostic and imaging agent. Because of the remarkable near-infrared optical features within the optimized light-transparent window of biomedical applications, ICG has been intensively studied for photodynamic therapy (PDT) and photothermal therapy (PTT) in tumor treatment.[37, 38] Singlet oxygen $\left({ }^{1} \mathrm{O}_{2}\right)$ is one of the highly reactive oxygen species which is believed to play a key role in the efficacy of PDT, an appropriate amount of ${ }^{1} \mathrm{O}_{2}$ from ICG is usually achieved by carrier encapsulation.[37] 
Herein, we developed a near-infrared light responsive-CDDP encapsulated synergistic therapy nanoplatforms for cancer treatment (Scheme 1). The CDDP and ICG were simultaneously loaded within the nanocarriers made from amphiphilic telluriumcontaining block copolymer PEG-PUTe-PEG. The coordination chemistry between platinum and tellurium guarantees a higher stability and prolonged circulation time in vivo. The tellurium atoms in the nanocarriers can be easily oxidized by ROS generated by ICG via the stimuli of a near-infrared laser. CDDP will be released from the nanocarriers as the oxidization of tellurium will weaken the coordination interaction with platinum. Selective drug release at target tissues can achieve high antitumor efficacy while significantly reduce side effects.[39-43]

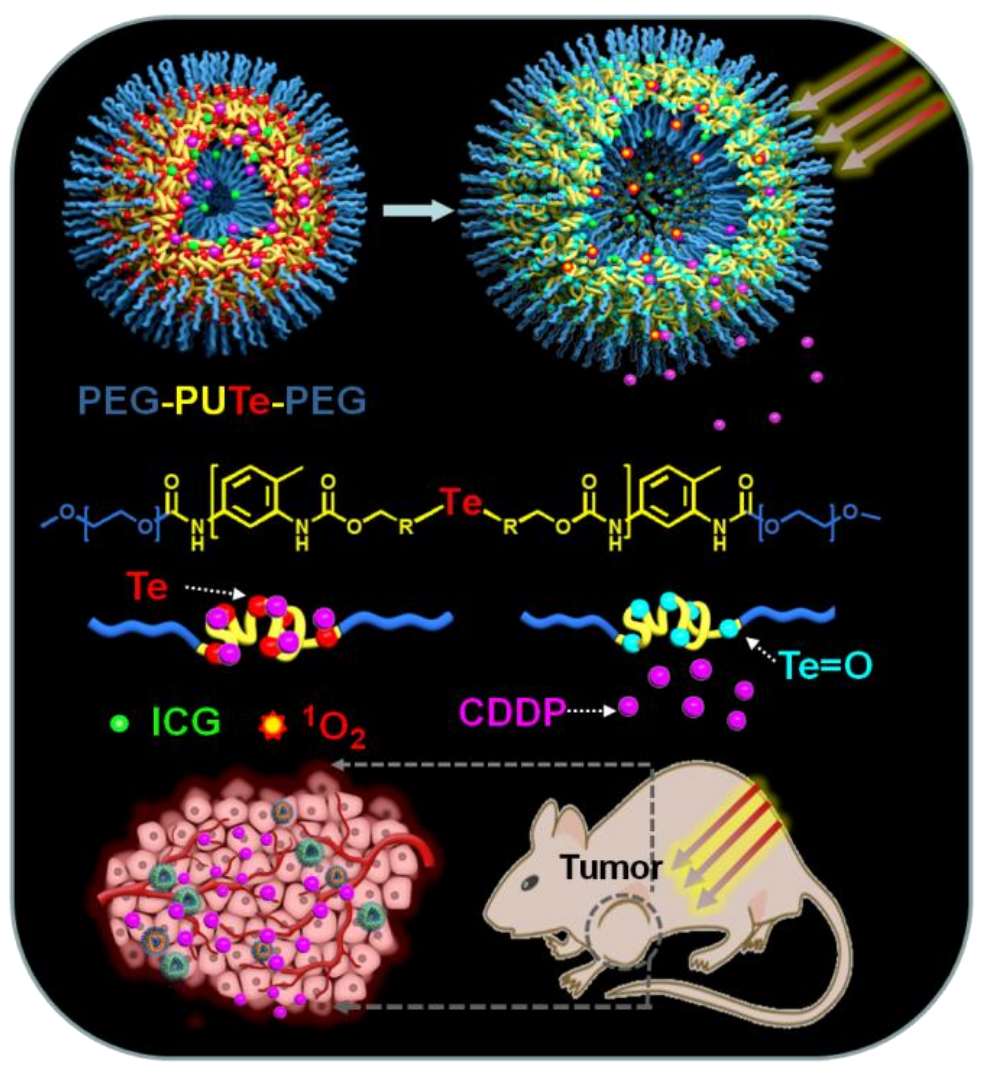


Scheme 1. Scheme of near-infrared light-stimuli CDDP release from amphiphilic polymer PEGPUTe-PEG based nanoparticles and the proposed blood circulation, drugs accumulation and release at tumor sites. The CDDP and ICG were loaded simultaneously in the multiple-layer nanoparticles made from PEG-PUTe-PEG. The nanoparticles have a prolonged circulation time in vivo as a contribution of the coordination chemistry between platinum and tellurium of PEGPUTe-PEG. Under the stimuli of a near-infrared laser at tumor sites, a amount of ROS is produced by ICG. The tellurium is easily oxidized by ROS because of the low electronegativity of tellurium and the CDDP could be rapidly released from the nanocarriers along with the oxidation of the tellurium at the tumor sites.

\section{Materials and methods}

\subsection{Materials and reagents.}

11-bromoundecanol, poly(ethylene glycol) $(\mathrm{PEG})$ monomethylether $(\mathrm{Mw}=5000)$, and CDDP were purchased from Sigma-Aldrich. Tellurium powder was obtained from Aladdin chemical company. Sodium borohydride, 2, 4-toluenediisocyanate (TDI) was product of Energy Chemical. All chemicals were used as received unless otherwise stated.

\subsection{Characterizations.}

${ }^{1} \mathrm{H}$ NMR spectra were recorded on a JEOL JNM-ECA 400 (400 MHz). For morphology characterization, nanoparticles were deposited on a carbon-coated copper grid, negatively stained with $2 \%$ uranyl acetate solution, and imaged with a transmission electron microscope (TEM, Tecnai G2 20 S-TWIN, FEI Ltd., USA). For 
dynamic light scattering (DLS) measurement, the size distribution of the PEG-PUTePEG based nanoparticles was measured using a Zetasizer Nano ZS90 (Malvern Instruments, Malvern, UK) with $90^{\circ}$ optics and a He-Ne Laser $(4.0 \mathrm{~mW}, 633 \mathrm{~nm})$. Xray surface photoelectron spectra were recorded on ESCALAB250Xi X-ray surface photoelectron spectroscopy (XPS). For inductively coupled plasma mass spectrometry measurement, an ELAN DRC-e ICP Mass Spectroscopy (ICP-MS, Perkin-Elmer, USA) was used. The electron paramagnetic resonance (EPR) spectra were performed on a Bruker ESP300E spectrometer operating at the X-band. For high performance liquid chromatography, an Agilent 1200 (The agilent technologies co., Ltd, USA) was used.

\subsection{Synthesis of PEG-PUTe-PEG Block Copolymer.}

First the telluride containing monomer Di-(1-hydroxylundecyl) telluride (denoted as $\mathrm{M}-\mathrm{Te}-\mathrm{OH}$ ) was synthesized through the reaction of disodium telluride and 11bromoundecanol. Disodium telluride was prepared through reaction between Te powder and excess amount of sodium borohydride at $50{ }^{\circ} \mathrm{C}$ in water under an atmosphere of argon. M-Te-OH was synthesized through the reaction of disodium telluride and 11-bromoundecanol in THF under $\mathrm{N}_{2}$ flow for $6 \mathrm{~h}$. Product obtained was purified by filtration and recrystallization. Then $1.34 \mathrm{~g}(1.35 \mathrm{mmol}) \mathrm{M}-\mathrm{Te}-\mathrm{OH}$ was dissolved in $10 \mathrm{~mL}$ of anhydrous THF in a $50 \mathrm{~mL}$ flask and was degassed by $\mathrm{N}_{2}$ for $20 \mathrm{~min}$. A solution of $0.42 \mathrm{~mL}(2.98 \mathrm{mmol})$ 2, 4-toluene diisocyanate (TDI) in $2 \mathrm{~mL}$ anhydrous THF was injected into the flask under $\mathrm{N}_{2}$ flow. The system was transferred 
into an oil bath at $50{ }^{\circ} \mathrm{C}$ to react for $12 \mathrm{~h}$ with stirring. $0.32 \mathrm{~g}(0.192 \mathrm{mmol})$ PEG (Mw $=5000$ ) was then dissolved in $4 \mathrm{~mL}$ of anhydrous THF and injected into the flask under $\mathrm{N}_{2}$ flow and the reaction was carried out for $12 \mathrm{~h}$. The solvent was then removed and the solid residue was washed three times by deionized water and acetone. A yellow powder of PEG-PUTe-PEG block copolymer was obtained after vacuum drying.

\subsection{Coordination of $\mathrm{M}-\mathrm{Te}-\mathrm{OH}$ with $\mathrm{CDDP}$.}

M-Te-OH was dissolved in DMSO-d6 together with cisplatin and kept still for different time before further characterization.

\subsection{Assembly of Nanoparticles.}

Nanoparticles were prepared by the double emulsion (W/O/W) method which have been reported in our previous work.[9] Briefly, $10 \mathrm{mg}$ of polymer PEG-PUTe-PEG was dissolved in $1 \mathrm{~mL}$ of methylene chloride (containing CDDP if required) and 0.2 $\mathrm{mL}$ of water (containing ICG if required). The mixture above was emulsified for 4 minutes by sonication. The emulsion was then mixed with $2 \mathrm{~mL}$ of $2 \%$ polyvinyl alcohol (PVA) and then emulsified for another 4 minutes by sonication. The emulsion was then slowly dropped into $10 \mathrm{~mL}$ of $0.6 \%$ PVA solution and stirred at room temperature for $10 \mathrm{~min}$. The organic solvent was removed by vacuum evaporation. The resulting nanoparticles were collected by centrifugation at room temperature and washed twice with ultrapure water. According to their different loading efficiencies 
(Figure S4), we controlled the amounts of CDDP and ICG added to the system in the final nanoparticle preparation depending on the needs of the experiments.

\subsection{Encapsulation Effciency and Drug Loading Content Calculations.}

Encapsulation efficiency of drugs in the NPs-Pt-ICG was calculated by the following equation. Encapsulation Efficiency $=(\mathrm{A}-\mathrm{B}) / \mathrm{A} \times 100 \%$, where the character $\mathrm{A}$ represents the initial amount of drug added in the system and B is the amount of drugs lefted in the supernatant after centrifugation of the nanoparticles. Drug loading content is calculated by the following equation. Loading Content $=(\mathrm{A}-\mathrm{B}) / \mathrm{C} \times$ $100 \%$, where A represents the initial mount of drug added in the system, B is the mount of drugs lefted in the supernatant of the after centrifugation and $\mathrm{C}$ represents the mount of the blank PEG-PUTe-PEG based nanoparticles. Inductively coupled plasma mass spectrometry and UV-visible absorption spectrum are utilized for quantification of CDDP and ICG respectively.

\subsection{Drugs release profiles.}

Inductively coupled plasma mass spectrometry and UV-visible absorption spectrum were used to determine the released amount of CDDP and ICG. The accumulative percentage of the released drugs was calculated as a function of incubation time. The in vitro release rates of drugs were calculated by the following equation: release rate $=$ $\mathrm{B} / \mathrm{A} \times 100 \%$, where $\mathrm{A}$ is the initial amount of drug encapculated in the system and $\mathrm{B}$ represents the amount of drugs obtained in the supernatant of the after centrifugation. The encapsulation effciency and drug loading content of CDDP in nanoparticles NPs- 
Pt-ICG were $54.13 \%$ and $9.97 \%$, respectively. The encapsulation effciency and drug loading content of ICG in nanoparticles NPs-Pt-ICG was $35.86 \%$ and $8.34 \%$, respectively.

\subsection{Photothermal Effects in Vitro.}

Aqueous solutions $(200 \mu \mathrm{L})$ of free ICG $(7.5 \mu \mathrm{M})$, NP-Pt, and NPs-Pt-ICG (7.5 $\mu \mathrm{M}$

ICG) were irradiated with an $808 \mathrm{~nm}$ continuous laser at a power density of $8 \mathrm{~W} / \mathrm{cm}^{2}$ and $1.4 \mathrm{~W} / \mathrm{cm}^{2}$ for $4 \mathrm{~min}$. The temperature was monitored every 10 seconds by a thermocouple.

\subsection{Cell Culture.}

Cell lines MDA-MB-231(a human breast carcinoma cell), HepG2 (a human liver carcinoma cell) and A549 (a human lung carcinoma cell) were cultured in Dulbecco's modified Eagle's medium (DMEM), supplemented with $10 \%$ fetal bovine serum at $37{ }^{\circ} \mathrm{C}$ in a humidified atmosphere containing $5 \% \mathrm{CO}_{2}$.

\subsection{Cytotoxicity Studies in Vitro.}

For in vitro cell viability assay, MDA-MB-231, HepG2 and A549 cells were seeded into 96-well plates at a density of 5000 cells per well. After seeding $12 \mathrm{~h}$, the cells were then co-incubated with various drug formulations for $24 \mathrm{~h}$. The cell viability was evaluated using a CCK-8 assay (Dojindo, Japan) according to the manufacturer's instructions.

\subsection{Imaging of Tumor Cells in Vitro.}


MDA-MB-231 cells were seeded at a density of $2 \times 105$ cells/well onto a $35 \mathrm{~mm}$ borosilicate chambered cover glasses (Nunc, USA.) and then cultured for $12 \mathrm{~h}$. The medium was then replaced with fresh medium containing different NPs formulations. After incubation at $37{ }^{\circ} \mathrm{C}$ for different times, the MDA-MB-231cells were washed three times with PBS and were imaged with an LSM 710 confocal microscope at 40x magnification.

\subsection{Fluorescent Images in Vivo.}

In vivo fluorescent images of $\mathrm{BALB} / \mathrm{c} \mathrm{nu} / \mathrm{nu}$ mice with tumor xenograft were measured using a IVIS lumina II in vivo spectrum imaging system at an excitation 710 nm.

\subsection{Xenograft Tumor Model in Nude Mice and in Vivo Antitumor Effect.}

To study the antitumor efficacy of the drug formulations in vivo, breast cancer tumor xenografts were formed in female BALB/C nu/nu mice by injecting MDA-MB-231 cells into the right flank (5 mice/group). When the tumor volume reached $60-70 \mathrm{~mm}^{3}$, various drug formulations (Saline, NPs, CDDP, NPs-Pt-ICG, NPs-ICG-Laser, and NPs-Pt-ICG-Laser) were injected through the tail vein on every three or four day thereafter. The laser treatment was carried out for a total of 9 min at a power density of $1.4 \mathrm{~W} / \mathrm{cm}^{2}$ with a 1 minute interval after every 2 minutes laser exposure $24 \mathrm{~h}$ after the drugs administration. Tumor sizes were measured using a traceable digital vernier caliper, and the tumor volumes were calculated using the formula $V=1 / 2 \mathrm{ab}^{2}$, where 
a represents the long axis and $\mathrm{b}$ is the short axis. Mice with tumors were euthanized after 26 days, and the tumor xenografts were excised for imaging and weighing.

\subsection{Analysis of the Side Effects of drug formulations in BALB/c Mice.}

To study the side effects of various drug formulations in vivo in our work, healthy female BALB/c mice were divided into five groups $(n=6)$ : saline control, CDDP, NPs, ICG and NPs-Pt-ICG. For evaluation of the potential side effect, the dose of CDDP and ICG were $6 \mathrm{mg} \mathrm{kg}^{-1}$ and $1.2 \mathrm{mg} \mathrm{kg}^{-1}$ respectively. Drug administrations were carried out on days 1,4 , and 7, and on day 13 the animals were euthanized and then various organs and serum were collected for further study. Serum was collected from three mice for biochemical tests in each group. The hearts, livers, and kidneys in group Saline, CDDP and NPs-Pt-ICG were fixed, sectioned and H \& E stained to study potential organ morphology changes.

\subsection{Statistical Analysis.}

SPSS version 18.0 software (SPSS Inc., Chicago, IL) was used for statistical analyses. The statistical differences between mean values were assessed by using student's ttest for unpaired data. Data are expressed as the mean \pm standard deviation (SD). A p value of less than 0.05 was considered statistically.

\section{Results and Discussion}

3.1 Synthesis of Te-containing ABA type Block Copolymer PEG-PUTe-PEG and Its Coordination Interaction with CDDP Study. 
The ABA type block copolymer PEG-PUTe-PEG was synthesized through a threestep reaction according to the method described in our previous work.[35] ${ }^{1} \mathrm{H}$ NMR measurement confirmed the successful synthesis of PEG-PUTe-PEG block copolymer (Figure 1) and the molecular weight $\left(\mathrm{M}_{\mathrm{n}}\right)$ was $83 \mathrm{k}$. The coordination interaction between tellurium and platinum was investigated by ${ }^{1} \mathrm{H}$ NMR and X-ray photoelectron spectroscopy (XPS) with tellurium-containing monomer, di-(1hydroxylundecyl) telluride (denoted as $\mathrm{M}-\mathrm{Te}-\mathrm{OH}$ ), as the model compound first. ${ }^{1} \mathrm{H}$ NMR characterizations (Figure 2A) showed that, 2.6 ppm peaks from the $\alpha$ protons of Te atom were gradually shifted to $2.9 \mathrm{ppm}$ after the complex formation. The XPS of $\mathrm{Te}^{3 \mathrm{~d}}$ showed that the binding energy of tellurium shifted upwards from 584.1 and $573.6 \mathrm{eV}$ to 584.9 and $574.5 \mathrm{eV}$ (Figure 2B), whereas the values for platinum slightly shifted downwards from 76.3 and $72.9 \mathrm{eV}$ to 76.1 and $72.7 \mathrm{eV}$ after the coordination process (Figure 2C), which was consistent with the results of ${ }^{1} \mathrm{H}$ NMR. The XPS data also demonstrated the further oxidation after treatment with low concentration $\mathrm{H}_{2} \mathrm{O}_{2}$ $(1 \%)$ as shown in Figure 2B. Interestingly, the binding energy of platinum shifted to the same value of CDDP after oxidized by $\mathrm{H}_{2} \mathrm{O}_{2}$ (Figure 2C), which indicated the disintegration of the complexation. 
M-Te-OH : ${ }^{\mathrm{HO}} \smile^{\mathrm{R}} \smile^{\mathrm{Te}} \smile^{\mathrm{R}} \smile_{\mathrm{OH}}^{\mathrm{OH}}=\mathrm{C}_{9} \mathrm{H}_{18}$

PEG-PUTe-PEG :

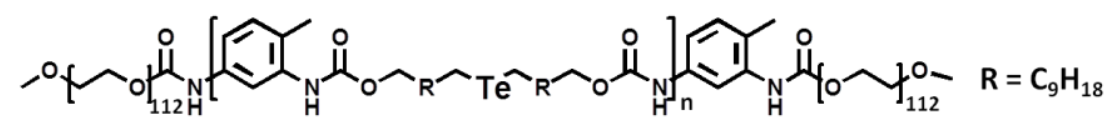

A

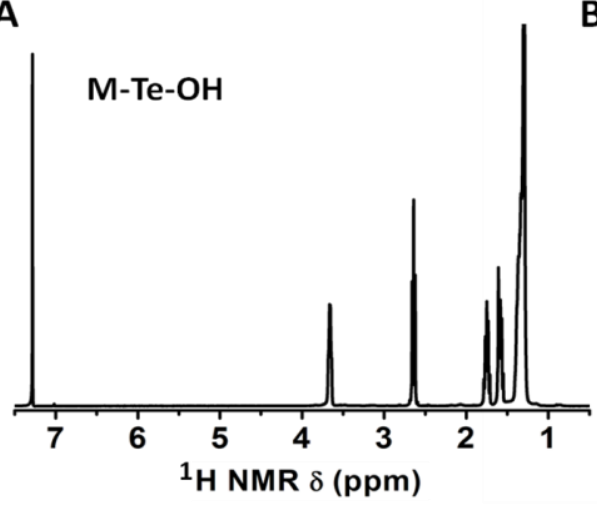

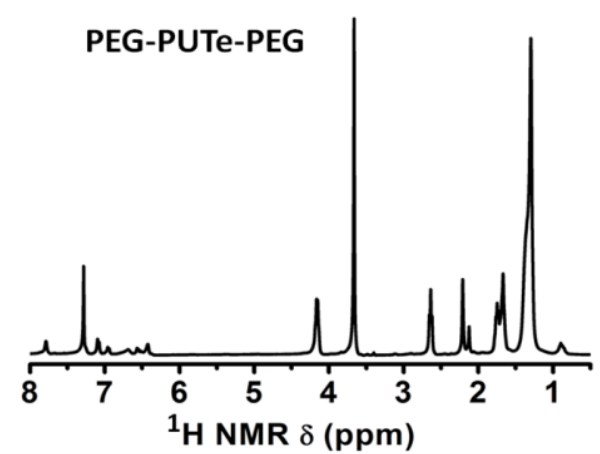

Figure 1. A) ${ }^{1} \mathrm{H}$ NMR spectrum of M-Te-OH (400MHz, CDCl3, $\left.298 \mathrm{~K}\right) \delta$ (ppm): 3.64 (4H, q, $\left.\left.\mathrm{HOCH}_{2}\right), 2.62\left(4 \mathrm{H}, \mathrm{t}, \mathrm{Te} \mathrm{CH}_{2}\right), 1.78-1.21\left(36 \mathrm{H}, \mathrm{m}, \mathrm{HOCH}_{2}\left(\mathrm{CH}_{2}\right)_{9} \mathrm{CH}_{2} \mathrm{Te}\right) ; \mathrm{B}\right){ }^{1} \mathrm{H}$ NMR spectrum of PEG-PUTe-PEG (400 MHz, CDCl3, $298 \mathrm{~K}) \delta(\mathrm{ppm}): 4.15\left(4 \mathrm{H}, \mathrm{b}, \mathrm{NHCOOCH}_{2}\right), 3.66(8 \mathrm{H}, \mathrm{b}$, $\mathrm{OCH}_{2} \mathrm{CH}_{2}$ of PEG), $2.62\left(4 \mathrm{H}, \mathrm{b}, \mathrm{Te} \mathrm{CH}_{2}\right), 2.18\left(4 \mathrm{H}, \mathrm{b}, \mathrm{TeCH}_{2} \mathrm{CH}_{2}\right), 1.80-1.21(32 \mathrm{H}, \mathrm{b}$, $\left.\mathrm{NHCOOCH}_{2}\left(\mathrm{CH}_{2}\right)_{8} \mathrm{CH}_{2} \mathrm{CH}_{2} \mathrm{Te}\right)$.
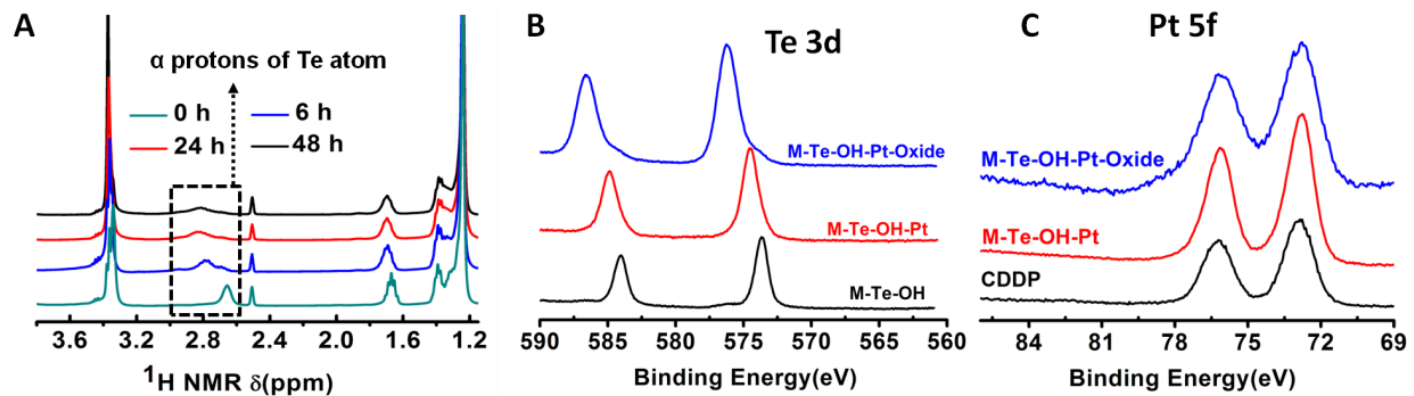

Figure 2. The coordination interaction of M-Te-OH with CDDP. A) ${ }^{1} \mathrm{H}$ NMR spectra of the MTe-OH in deuterated dimethylsulfoxide with CDDP for different time. The $\alpha$ protons of Te atom.

B) The deconvoluted XPS spectra of $\mathrm{Te}^{3 \mathrm{~d}}$ in $\mathrm{M}-\mathrm{Te}-\mathrm{OH}, \mathrm{M}-\mathrm{Te}-\mathrm{OH}-\mathrm{Pt}$ (coordination compound of $\mathrm{M}-\mathrm{Te}-\mathrm{OH}$ and $\mathrm{CDDP}$ ), and $\mathrm{M}-\mathrm{Te}-\mathrm{OH}-\mathrm{Pt}-$ oxide (oxidized $\mathrm{M}-\mathrm{Te}-\mathrm{OH}-\mathrm{Pt}$ by $\mathrm{H}_{2} \mathrm{O}_{2}$ ). C) The deconvoluted XPS spectra of $\mathrm{Pt}^{5 \mathrm{f}}$ in CDDP, M-Te-OH-Pt, and M-Te-OH-Pt-oxide. 


\subsection{Preparation of PEG-PUTe-PEG Based Nanoparticles.}

We then prepared the nanoparticles through a double emulsion method.[44] In this method, CDDP was mainly packaged within the hydrophobic layer of the nanoparticles based on the coordination interaction between tellurium and platinum, while ICG was inserted into both the hydrophobic layer and hydrophilic core (Scheme 1). We confirmed the successful encapsulation of ICG in NPs using UV-vis absorbance spectra (Figure 3A) as the drugs-loaded nanoparticles (NPs-Pt-ICG) had the characteristic absorption bands of ICG with a slightly red shift, which is a contribution of the aggregation of ICG.[37,38] Unfortunately, the UV-vis absorption of CDDP is not easily detected due to the strong absorption of the unloaded nanoparticles (NPs) in the ultraviolet region. Then high performance liquid chromatography (HPLC) spectra were used to confirm the successful encapsulation of CDDP (Figure 3B). The characteristic absorption band of CDDP was not detected in the supernatant of NPs-Pt-ICG. Interestingly, it was detected in the supernatant of oxidized NP-Pt-ICG by $\mathrm{H}_{2} \mathrm{O}_{2}$ and NPs-Pt-ICG-Laser (oxidized NPs-Pt-ICG by laser exposure). These results also demonstrated the ROS-stimuli CDDP release property of NPs-Pt-ICG. The successful CDDP encapsulation was also detected using XPS as shown in the Figure 3C. The binding energy of tellurium shifted upwards with the coordination interaction happened. However, the ${ }^{1} \mathrm{O}_{2}$ produced by ICG in the NPs-PtICG (Figure S1, Supporting Information) can further oxidize the tellurium in the nanoparticles which can induce the CDDP release (Figure 3B). 

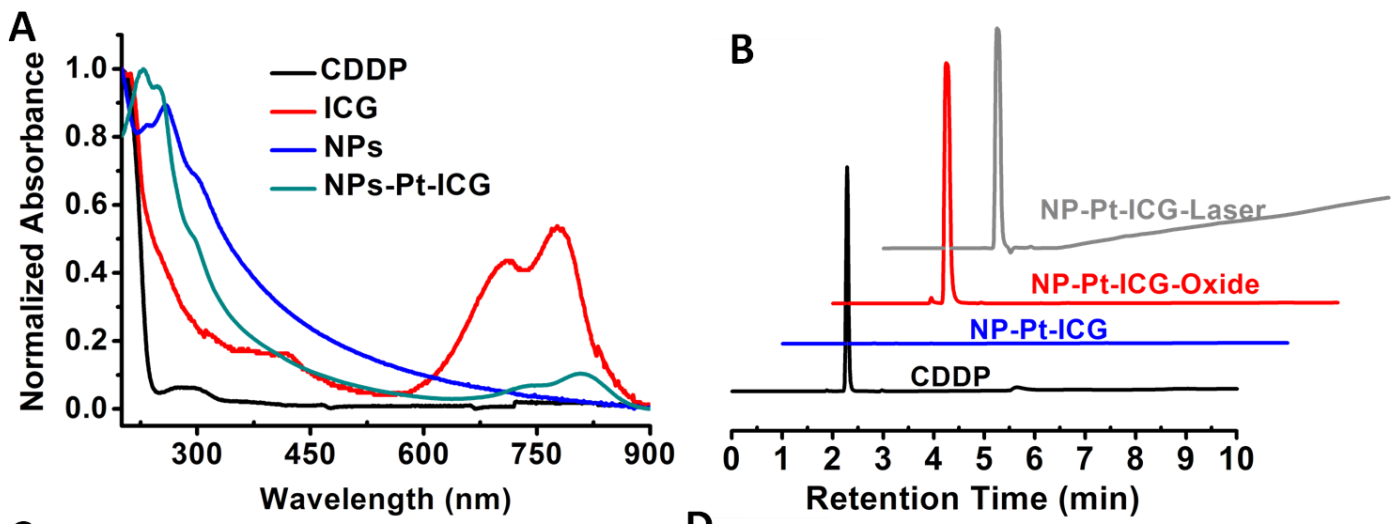

C

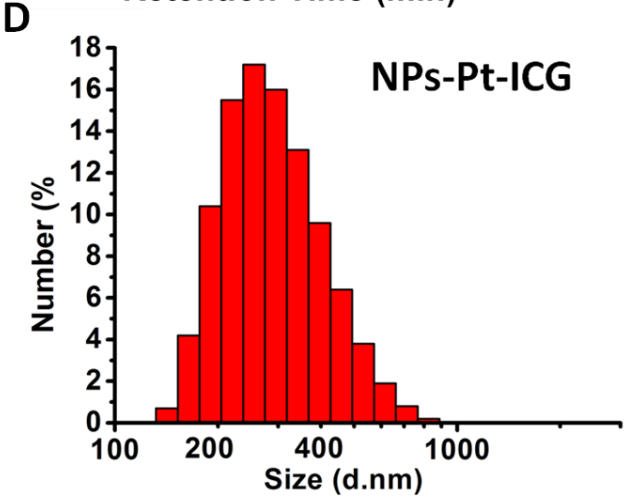

Figure 3. Characterization of PEG-PUTe-PEG Based CDDP and ICG loaded Nanoparticles. A)UV-vis absorbance spectra of CDDP, ICG, NPs, and NPs-Pt-ICG. B) The HPLC measurement of free CDDP, the supernatant of NPs-Pt-ICG, NPs-Pt-ICG-Oxide (oxidized NPs-Pt-ICG by $\mathrm{H}_{2} \mathrm{O}_{2}$ ) and NPs-Pt-ICG-Laser (oxidized NPs-Pt-ICG by laser exposure) C) The deconvoluted XPS spectra of the $\mathrm{Te}^{3 \mathrm{~d}}$ in NPs, NPs-Pt-ICG and NPs-Pt-ICG-Laser (near infrared laser induced oxidation of NPs-Pt-ICG). D) The size distributions of NPs-Pt-ICG measured by DLS

The size and stability property of NPs are important factors for drug delivery. The size distribution of NPs-Pt-ICG was characterized by dynamic light scattering (DLS). The results indicated the size distribution of NPs-Pt-ICG (Figure 3D) was a little smaller than that of NPs (Figure S2). The stability of NPs-Pt-ICG was investigated by dispersing the nanoparticles in $10 \%$ fetal bovine serum (FBS) at room temperature and examining the size of the particles by DLS over time (Figure S3). There was no 
significant change in the size distribution of NPs-Pt-ICG even after $72 \mathrm{~h}$ incubation, which implies the good stability of the NPs-Pt-ICG in serum. However, the NPs treated in the same way were of a broader particle size distribution (data not show). The coordination interaction between tellurium and platinum may have significant contribution to the good stability.

\subsection{Drug release profiles and the morphology change with the light exposure.}

The high drug loading and controlled release of drugs are advantages of drug-loaded nanoparticles as they can improve bioavailability and reduce the toxicity of drugs to normal tissues. The drug encapsulation yields were investigated (Figure S4), and the results showed the NPs were effective for co-delivery ICG and CDDP, and the highest encapsulation efficiency and loading content of CDDP were $69.79 \%$ and $30.62 \%$, respectively. The release profiles of NPs-Pt-ICG in vitro were obtained at room temperature (Figure 4A and B). NPs-Pt-ICG lost only a small proportion (less than $20 \%$ ) of its cargoes over 5 days without laser exposure as shown in Figure 4A. However, within 10 minutes, most of the CDDP in the nanoparticles could be released with the laser $(808 \mathrm{~nm})$ exposure, as shown in Figure 4B. The morphological transformation of NPs-Pt-ICG with laser exposure was characterized by TEM (Figure 4C). The initial nanoparticles were dispersed, with a well-defined spherical structure, and an average diameter at approximately $200 \mathrm{~nm}$ (Figure 4C (a)). However, after the laser exposure, obvious core-shell multilayer structure generated along with the oxidation of the tellurium on the polymer backbone by ${ }^{1} \mathrm{O}_{2}$ produced by ICG. The 
core expanded gradually but the shell tended to shrink with the progressing of oxidation. And the hydrodynamic size of NPs-Pt-ICG became much lager after NIR irradiation (d) (Figure 3D and Figure S5). We speculate that the oxidation of tellurium makes the hydrophobic block of the polymer more hydrophilic, [30, 31] which facilitates the free diffuse of water in and out of the nanoparticle's core. The change of chemical property and structure in the aggregates was consistent with the fast CDDP release from the nanoparticles.

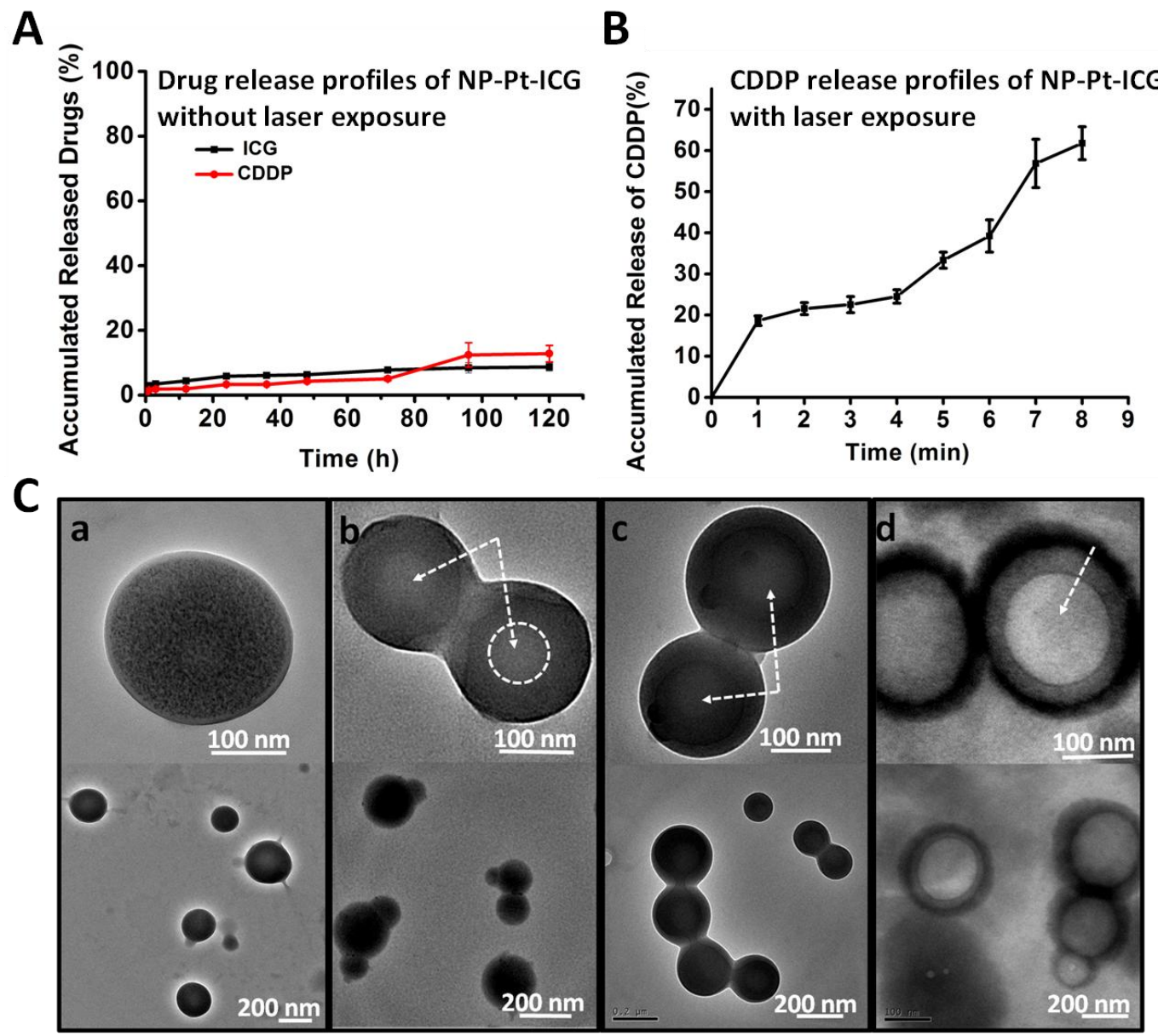

Figure 4. Drug release profiles and morphology changes with the light exposure $(808 \mathrm{~nm})$ prolonged. A) Drugs release profiles of NPs-Pt-ICG at room temperature (n=3). B) CDDP release 
profiles of NPs-Pt-ICG with near-infrared laser exposure $(n=3)$. C) Morphology change with the near-infrared laser exposure (a, 0 min; b, 1 min; c, 3 min; d, 7min) characterized by TEM.

\subsection{Cellular Uptake and Intracellular Distribution}

One prerequisite for efficient drug delivery is high nanomedicine uptake level. To investigate this, we encapsulated in the NPs-Pt (NPs loaded with CDDP) with a green dye fluorescein isothiocyanate (FITC) and red dye rhodamine B (RhoB) (NPs-PtFITC-RhoB). MDA-MB-231 cells were incubated with NPs-Pt-FITC-RhoB and then examined at various time points by confocal microscopy to determine the intracellular distribution of the fluorescent compounds. The fluorescence spectra of NPs-Pt-FITC and NPs-Pt- RhoB demonstrated that the they had similar fluorescence emission character to the free dyes (Figure 6S). As shown in Figure 5A, the cellular fluorescent intensity was enhanced over time, suggesting that the NPs-Pt-FITC-RhoB were internalized continuously. The major fluorescence signal was orange, consistent with the overlapping fluorescence of the red and green, indicating most NPs-Pt-FITCRhoB maintained integrated even 3 hours after cellular uptake. However, drugs must be released into the cytoplasm to exert their function. As illustrated in Figure 5B, after $3 \mathrm{~h}$ incubation with NPs-Pt-FITC, separation of green (FITC) and red (LysoTracker Red stained lysosome) fluorescence signals demonstrated that most of the NPs-PtFITC were located in cytoplasm after endocytosis rather than degraded in the lysosomes. 


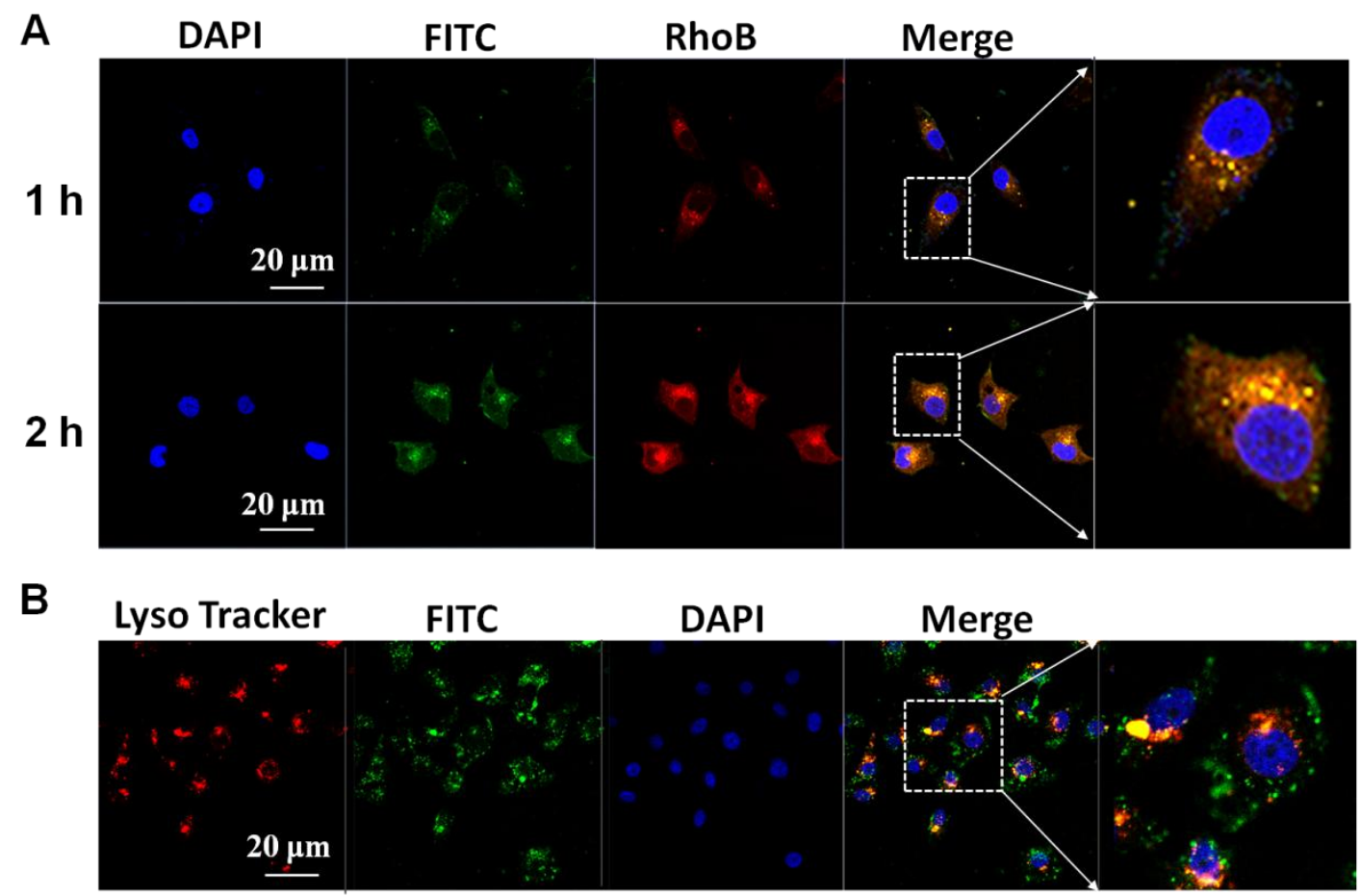

Figure 5. Cellular uptake, intracellular distribution of nanoparticles. A) Cellular uptake of NPs-Pt.

Confocal microscopic images of MDA-MB-231cells treated with NPs-Pt-FITC-RhoB at different time points. Cell nucleuses (blue) were stained by DAPI. B) Intracellular distribution of NPs-PtFITC. The lysosomes were stained with LysoTracker Red.

\subsection{Cytotoxicity Study in Vitro.}

Having established that NPs-Pt-ICG were able to effectively delivery CDDP into cells, we needed to determine whether the unloaded tellurium-containing NPs have cytotoxicity to cells. A549, HepG2 and MDA-MB-231 cell lines were co-incubated with NPs at different concentrations for $24 \mathrm{~h}$, followed by quantification of cell viability using the CCK-8 assay. The results (Figure 6A) demonstrated that the blank NPs exhibited almost no cytotoxicity to different cell lines. The cytotoxicity of NPsICG and NPs-ICG-Oxide (oxidized NPs-ICG by laser exposure) was also investigated 
in the same way with MDA-MB-231 cell line and no cytotoxicity to the cells has been observed, as shown in Figure 6B.

A

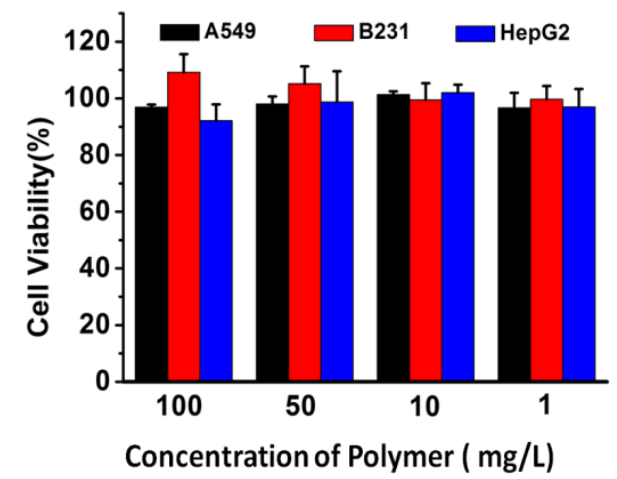

B

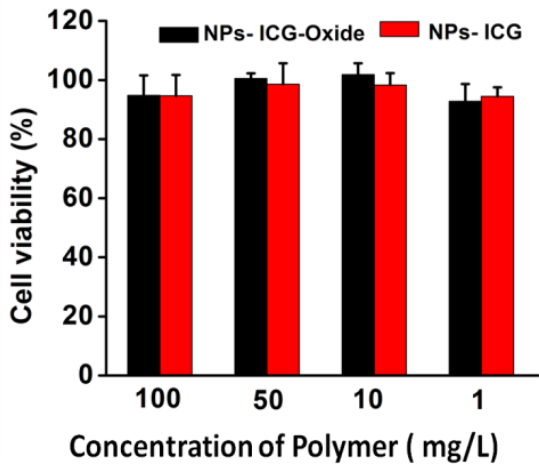

Figure 6. Cytotoxicity of different nanoparticle formulations in vitro. A) Cytotoxicity of NPs in vitro. Viability of MDA-MB-231, A549 and HepG2 cells after exposure to different concentrations of blank NPs for $24 \mathrm{~h}$. B) Cytotoxicity of NPs-ICG and NPs-ICG-oxide (light induced oxidation of NPs-ICG). Viability of MDA-MB-231 cells after exposure to different concentrations of nanoparticles for $24 \mathrm{~h}$. In A) and B) the cell survival of no treatment was set as $100 \%$.

\subsection{Circulation Time and Tumour Targeting in Vivo}

To investigate whether the CDDP loaded nanoparticles have prolonged circulation time in vivo, SD rats with jugular vascular catheterizations were separated into two groups and injected intravenously with free CDDP and NPs-Pt-ICG, respectively. Blood samples were collected from the catheters at various time points after the administration. Free CDDP disappeared rapidly from the blood circulation with reduction more than $90 \%$ within $1 \mathrm{~h}$, while more than $15 \%$ of platinum or tellurium of NPs-Pt-ICG still remained $24 \mathrm{~h}$ after the administration (Figure 7A). These data 
suggested that NPs and NPs-Pt-ICG had a prolonged circulation time in vivo, a desirable characteristic for enhanced nanoparticles accumulation at tumor sites.

To investigate whether the NPs-Pt-ICG could target tumors in vivo, we established a subcutaneous tumor-bearing mouse model. Tumor-bearing mice were sorted into three groups and injected with saline, free ICG, and NPs-Pt-ICG, respectively. Fluorescence signals were recorded at 1, 6, and $24 \mathrm{~h}$ after injection with an IVIS lumina II in vivo optical imaging system. As shown in Figure 7B, free ICG initially distributed throughout the body, but they were rapidly degraded and/or cleared. NPsPt-ICG tended to accumulate at the site of the tumors due to enhanced permeability and retention (EPR) effect. After $24 \mathrm{~h}$, mice were sacrificed, and organs and tumors were collected for ex vivo fluorescence imaging. Fluorescence signals in organs and tumor of free ICG group were very weak. In NPs-Pt-ICG group, the fluorescence signals were much stronger, with most signals concentrated at tumor tissues. These results indicated that NPs-Pt-ICG preferentially accumulated at the site of the tumor relative to free ICG. This maybe a contribution of their prolonged circulation time in vivo.

A

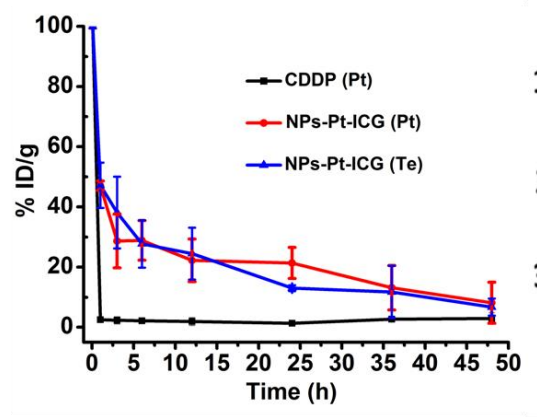

B

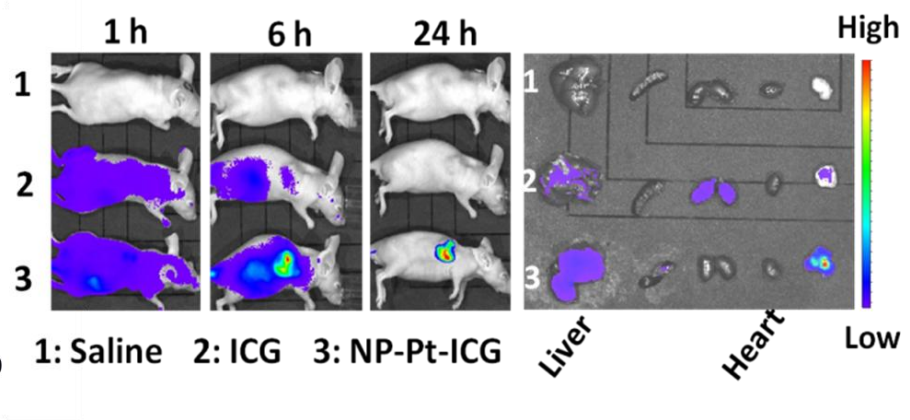


Figure 7. Prolonged circulation time and targeting effects in vivo in subcutaneous tumor model. A) The circulation time of CDDP and NPs-Pt-ICG. Blood samples were drawn from the rats after injection with free CDDP and NPs-Pt-ICG, respectively. The platinum and tellurium concentrations were measured with inductively coupled plasma mass spectrometry. The concentration of $5 \mathrm{~min}$ in each group was normalized as $100 \%$. Data are expressed as mean \pm S.D. $(\mathrm{n}=3)$. B) In vivo targeting of NPs-Pt-ICG. BALB/c nude mice with subcutaneous xenografts (MDA-MB-231 cells) were injected through tail vein with free ICG and NPs-Pt-ICG. After $24 \mathrm{~h}$, the mice were sacrificed, and the tumor, kidney, liver, lung, spleen and heart were removed for $e x$ vivo fluorescence imaging.

\subsection{Antitumor Effects in Vivo.}

To investigate whether the near-infrared light-mediated CDDP encapsulated synergistic therapy nanoplatforms could enhance antitumor effects in vivo, we took BALB/c nu/nu mice with MDA-MB-231 subcutaneous xenografts and divided them into six groups. We began the intravenous injection of the drugs (or saline) as the arrows indicated when the tumors reached a predetermined size in Figure 8A. Although NPs-Pt-ICG has excellent photothermal conversion efficiency in vitro (Figure S7 and Figure S8), to avoid possible tissue damage by hyperthermia, the laser treatment was carried out for a total of $9 \mathrm{~min}$ at a power density of $1.4 \mathrm{~W} / \mathrm{cm}^{2}$ with a 1 min interval after every 2 min of exposure about $24 \mathrm{~h}$ after the drugs administration. Tumors grew progressively and rapidly in the saline and the NPs group (Figure 8A). The unencapsulated CDDP can inhibit the growth of tumors effectively, though its 
serious side effects caused severe emaciation and even death (two mice died after four times treatments, and drug administration had to be stopped considering their physical conditions). NPs-Pt-ICG reduced tumor growth to a certain degree. As a contribution of photothermal effect of ICG, NPs-ICG-Laser group showed a modest slower growth compared with the saline group. However, the most effective repression of cancer progression was observed in the NPs-Pt-ICG-laser group as shown in Figure 8A and B. In this group the tumors of two mice disappeared completely. Consistent with the growth curves and tumor images, the mean weight of the tumors in the NPs-Pt-ICGlaser group was the lowest of all the treatments. H\&E staining was used to further validate the extent of apoptosis in the tumors after treatment with various drug formulations (Figure 8C). In the groups treated with Saline and NPs, only basal level apoptosis was seen. Apoptosis with different degree can be found in the groups treated with CDDP, NP-Pt-ICG and NP-ICG-Laser, respectively. However, the most serious apoptosis was found in the group treated with NPs-NP-ICG-Laser. These results demonstrated that NPs-Pt-ICG is able to further enhance the antitumor effects of CDDP under laser exposure. We hypothesize the near-infrared light can induce rapid and efficient release of the encapsulated CDDP in NPs-Pt-ICG at tumor sites and the released CDDP easily diffuse into tumors compared with NPs-Pt-ICG which contribute better antitumor effect. In addition, the thermotherapy effect also displayed a synergistic antitumor effect. 

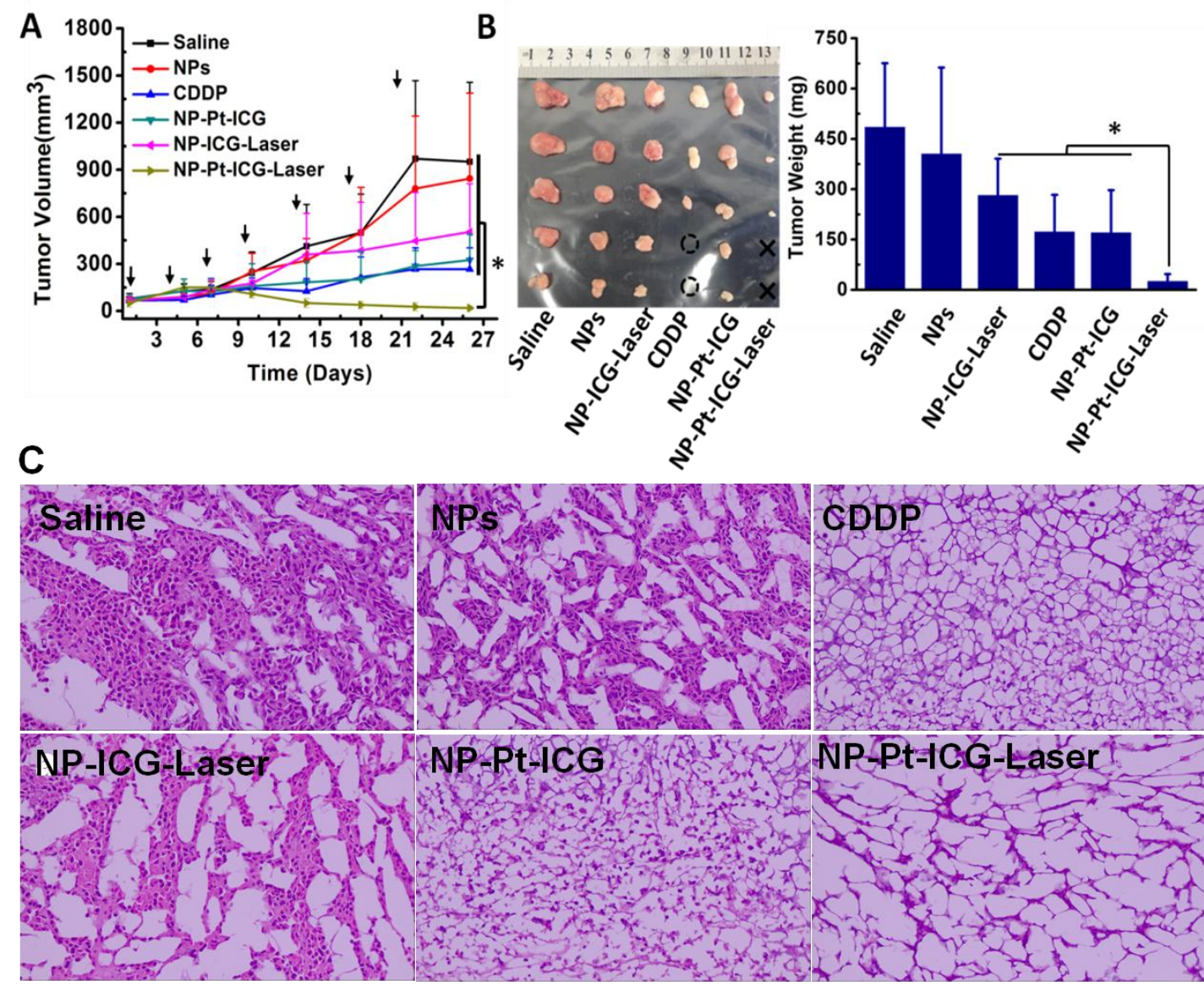

Figure 8. Antitumor effects in subcutaneous tumor model. MDA-MB-231 cells were subcutaneously injected into BALB/c nude mice. A) Tumor growth curves. B) When treatment was finished, the tumor xenografts were excised, and the tumor weights were obtained. $* \mathrm{P}<0.05$.

The black circles indicated dead mice and the black cross indicated disappeared tumors. C) H\&E staining for apoptotic cells in tumor xenografts after treatments with different drug formulations.

\subsection{Side Effects Study of NPs-Pt-ICG in Vivo}

Effects of the drug preparations on healthy BALB/c mice with normal immunity was examined to determine whether NPs-Pt-ICG could decrease the side effect of CDDP. Five groups were studied: saline control, CDDP, NPs, ICG and NPs-Pt-ICG $(n=6)$. Only mice injected with free CDDP lost a significant amount of weight as shown in

Figure 9A. Serum biochemical tests were carried out to further appraise the potential 
effects of these drug preparations on the heart, liver, and kidney. Free CDDP administration significantly changed the biochemical indicators in the liver, heart, and kidney compared to saline group (Figure 9B ad Figure S9). These data suggested that the biological functions of the organs of CDDP administration mice were subjected to severe damage or dysfunction. On the contrary, the NPs-Pt-ICG treated group displayed negligible differences in liver function, myocardial enzyme spectrum, and renal function tests compared with the saline group, indicating that these organs were restored to near normal conditions. The results of ICG and NPs groups demonstrated they had little influence on the functions of the organs.

The postmortem histopathology of the heart, liver, and kidney were analyzed to study potential changes in organ morphology (Figure 9C). Compared with other four groups, infiltration of inflammation cell and local renal tubular and glomerular involvement in the CDDP treated group can be easily observed as most CDDP is excreted rapidly from the kidney after administration. In addition, a small amount of inflammation cells in liver and vacuolation in heart suggested a certain degree of damage of the organs in the CDDP treated group. Fortunately, no obvious morphological changes were observed in NPs-Pt-ICG treated group. These data suggested that the severe organ damage, especially in kidney, associated with free CDDP administration was efficiently alleviated by encapsulation in the NPs. 

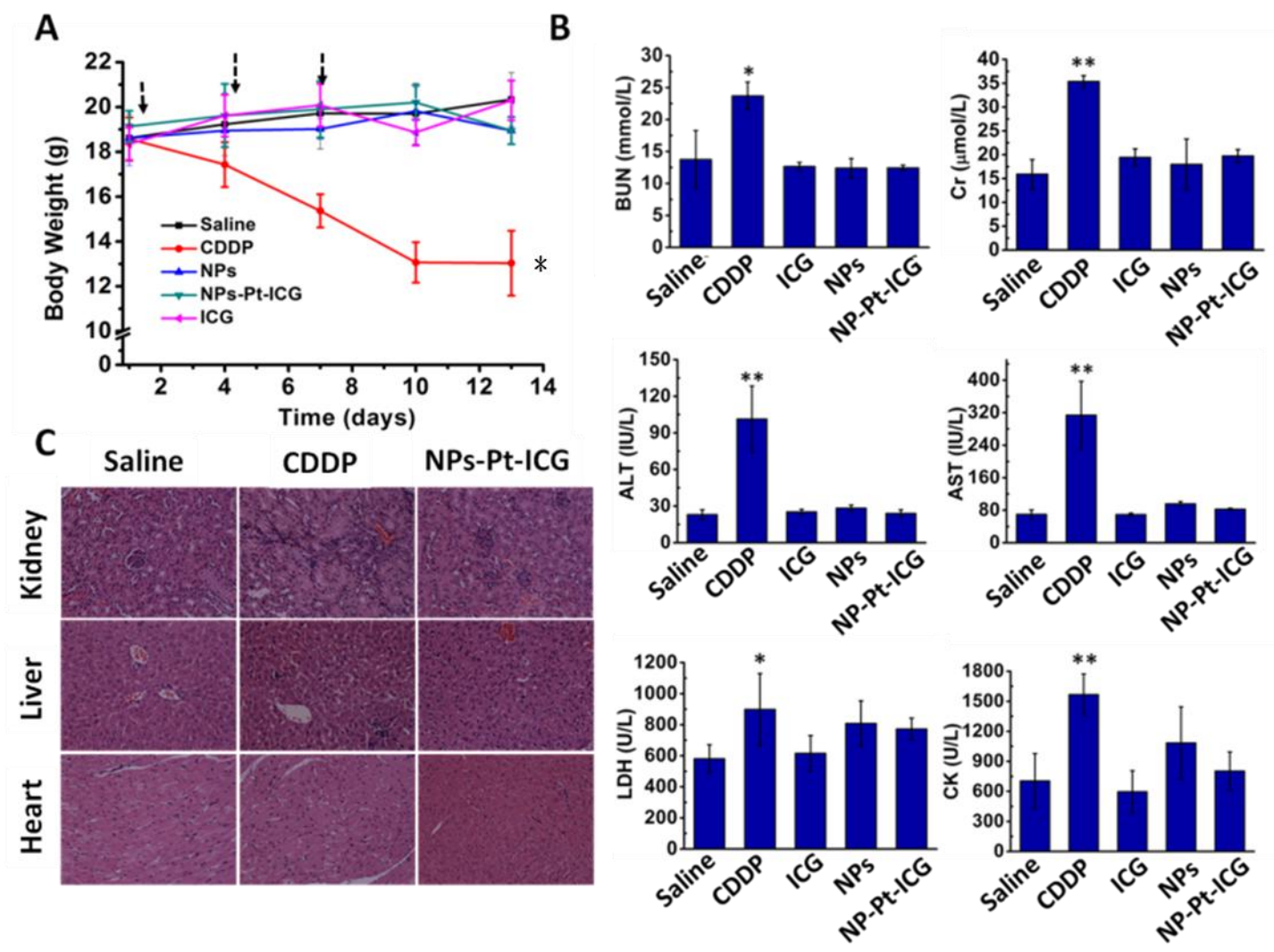

Figure 9. Evaluation of potential adverse effects of various drug formulations. A) Body weight of various treated mice as a function of time. $* \mathrm{P}<0.05$ vs saline. B) Serum biochemical tests results $(\mathrm{n}=3)$. Data are presented as mean \pm S.D. $* \mathrm{P}<0.05$ vs saline. ${ }^{* *} \mathrm{P}<0.01$ vs saline. ALT, alanine transaminase; AST, aspartate transaminase; BUN, blood urea nitrogen; $\mathrm{Cr}$, creatinine; LDH, lactic dehydrogenase; CK, creatine kinase. C) Representative H\&E staining of various organs.

\section{Conclusion}

In summary, by employing the coordination chemistry between platinum and tellurium, we developed a CDDP drug delivery system with near-infrared lightstimuli drug release property by encapsulating CDDP and ICG in the novel telluriumcontaining polymer (PEG-PUTe-PEG) based nanoparticles. The obtained vehicle 
achieves both promising antitumor effects and minimal side effects on the major organs in combination with the photothermal effect of ICG. The nanoparticles display a high stability that can afford prolonged circulation time in vivo due to the coordination chemistry between platinum and tellurium, and the rapid CDDP release can be realized with the stimuli of a near- infrared laser at tumor sites leading to the oxidation of the tellurium in the nanoparticles, facilitating drug penetration into the tumor microenvironment. The work lays the foundation for the further development of CDDP delivery synergistic therapy nanoplatform for cancer therapy.

\section{Competing financial interests}

The authors declare no competing financial interests.

\section{Author contributions}

F.L. and H.X. designed the project. F.L., W.C. and L.W. prepared the polymer material. F.L. and T.L. performed the experiments, analysed the data, and wrote the paper under the supervision of H.X. All authors discussed the progress and reviewed the manuscript.

\section{Acknowledgements}

This work was supported by the National Basic Research Program of China (2013CB834502), the National Science Foundation for Distinguished Young Scholars (21425416), the Foundation for Innovative Research Groups of the National Natural Science Foundation of China (21421064), the Natural Science Foundation of Beijing for Young Scholars (041560241), and the China Postdoctoral Science Foundation (043260277). All procedures for animal use were approved by the Institutional 
Animal Care and Use Committee (IACUC) at Tsinghua University with assurance number \#A5916-01, and all efforts were made to minimize animal suffering. The authors acknowledge Guangjun Nie and Yutao Yin et al. for their help in animal experiments.

\section{Appendix A. Supplementary data}

Supplementary data related to this article can be found at $\mathrm{xxxxx}$

\section{References}

1 A.S. Abu-Surrah, M. Kettunen, Platinum group antitumor chemistry: design and development of new anticancer drugs complementary to cisplatin, Curr. Med. Chem. 13 (2006) 1337-1357.

2 L. Kelland, The resurgence of platinum-based cancer chemotherapy, Nat. Rev. Cancer 7 (2007) 573-584.

3 X.J. Liang, H. Meng, Y. Wang, H. He, J. Meng, J. Lu, P.C.Wang, Y. Zhao, X. Gao, B. Sun, Metallofullerene nanoparticles circumvent tumor resistance to cisplatin by reactivating endocytosis, Proc. Natl. Acad. Sci. U.S.A. 107 (2010) 7449-7454.

4 R.S. Go, A.A. Adjei, Review of the comparative pharmacology and clinical activity of cisplatin and carboplatin, J. Clin. Oncol. 17 (1999) 409-422.

5 S.R. McWhinney, R.M. Goldberg, H.L. McLeod. Platinum neurotoxicity pharmacogenetics, Mol. Cancer. Ther. 8 (2009) 10-16.

6 C.A. Rabik, M.E. Dolan, Molecular mechanisms of resistance and toxicity associated with platinating agents, Cancer Treat. Rev. 33 (2007) 9-23. 
7 D. Peer, J.M. Karp, S. Hong, O.C. FaroKHzad, R. Margalit, R. Langer, Nanocarriers as an emerging platform for cancer therapy, Nat. Nanotechnol. 2 (2007) 751-760.

8 T.M. Sun, Y.S. Zhang, B. Pang, D.C. Hyun, M.X. Yang, Y.N. Xia, Engineered nanoparticles for drug delivery in cancer therapy, Angew. Chem. Int. Ed. 53 (2014)12320-12364.

9 X. Zhao, F. Li, Y.Y. Li, H. Wang, H. Ren, J. Chen, G.J. Nie, J.H. Hao, Codelivery of HIF1 $\alpha$ siRNA and gemcitabine via biocompatible lipid-polymer hybrid nanoparticles for eEffective treatment of pancreatic cancer, Biomaterials $46(2015) 13-25$.

10 H.P. Xu, W. Cao, X. Zhang, Selenium-containing polymers: promising biomaterials for controlled release and enzyme mimics, Acc. Chem. Res. 46 (2013) 1647-1658.

11 W Cao, Y.W. Gu, M. Meineck, H.P. Xu, The combination of chemotherapy and radiotherapy towards more efficient drug delivery, Chem. Asian. J. 9 (2014) 4857.

12 I.C. Kwon, Y.H. Bae, S.W. Kim, Electrically credible polymer gel for controlled release of drugs, Nature 354 (1991) 291-293.

13 X.L. Hu, G.H. Liu, Y. Li, X.R. Wang, S.Y. Liu, Cell-penetrating hyperbranched polyprodrug amphiphiles for synergistic reductive milieu-triggered drug release 
and enhanced magnetic resonance signals, J. Am. Chem. Soc. 137 (2015) 362368.

14 LY. Li, K. Raghupathi, CH. Yuan, Surface charge generation in nanogels for activated cellular uptake at tumor-relevant pH, Chem. Sci. 4 (2013) 3654-3660.

15 J. Kim, S. Pramanick, D. Lee, H. Park, W.J. Kim, Polymeric biomaterials for the delivery of platinum-based anticancer drugs, Biomater. Sci. 3 (2015) 1002-1017.

16 H.C. Chen, W.J. He, Z.J. Guo, An $\mathrm{H}_{2} \mathrm{O}_{2}$-responsive nanocarrier for dual-release of platinum anticancer drugs and O-2: controlled release and enhanced cytotoxicity against cisplatin resistant cancer cells, Chem. Commun. 50 (2014) 9714-9717.

17 A.P.A. Favareto, L.C. Nogueira, D.A. Pontes, S.D.P. Mesquita, I.C.C. Camargo, Evaluation of Testicular Tissue of Adult Rats Treated With Cisplatin Incorporated Into the Liposome, Microsc. Res. Tech. 78 (2015) 323-329.

18 Y.Z. Min, J.M. Li, F. Liu, E.K.L. Yeow, B.G. Xing, Near-infrared light-mediated photoactivation of a platinum antitumor prodrug and simultaneous cellular apoptosis imaging by upconversion-luminescent nanoparticles, Angew. Chem. Int. Ed. 53 (2014) 1012-1016.

19 L. Tang, R. Tong, V.J. Coyle, Q. Yin, H. Pondenis, L.B. Borst, J. Cheng, T.M. Fan, Targeting tumor vasculature with aptamer-functionalized doxorubicinpolylactide nanoconjugates for enhanced cancer therapy, ACS Nano 9 (2015) $5072-5081$ 
20 K.J. Haxton, H.M. Burt, Polymeric drug delivery of platinum-based anticancer agents, J. Pharm. Sci. 98 (2009) 2299-2316.

21 N. Nishiyama, S. Okazaki, K. Kataoka, Novel cisplatin-incorporated polymeric micelles can eradicate solid tumors in mice, Cance Res. 63 (2003) 8977-8983.

22 M. Baba, Y. Matsumoto, A. Kashio, H. Cabral, N. Nishiyama, K. Kataoka, Y.T. Amasoba, Micellization of cisplatin (NC-6004) reduces its ototoxicity in guinea pigs, J. Control. Release 157 (2012) 112-117.

23 R. Plummer, R.H. Wilson, H. Calvert, A.V. Boddy, M. Griffin, J. Sludden, M.J. Tilby, M. Eatock, D.G. Pearson, C.J. Ottley, Y. Matsumura, K. Kataoka, T. Nishiya, A phase I clinical study of cisplatin-incorporated polymeric micelles (NC-6004) in patients with solid tumors, Br. J. Cancer 104 (2011) 593-598.

24 B. Schechter, A. Neumann, M. Wilchek, R. Arnon, Soluble polymers as carriers of cis-platinum, J. Control Release 10 (1989) 75-87.

25 L.Y. Liao, J. Liu, E.C. Dreaden, S.W. Morton, K.E. Shopsowitz, P.T. Hammond, J.A. Johnson, A convergent synthetic platform for single-nanoparticle combination cancer therapy: ratiometric loading and controlled release of cisplatin, doxorubicin, and camptothecin, J. Am. Chem. Soc. 136 (2014) 5896-5899.

26 V.T. Huynh, J.Y. Quek, P.L. de Souza, M.H. Stenzel, Block copolymer micelles with pendant bifunctional chelator for platinum drugs: effect of spacer length on the viability of tumor cells, Biomacromolecules 13 (2012) 1010-1023. 
27 L.E. Gerweck, S. Vijayappa, S. Kozin, Tumor $\mathrm{pH}$ controls the in vivo efficacy of weak acid and base chemotherapeutics, Mol. Cancer. Ther. 5 (2006) 1275-1279.

28 J. Zhuang, M. Gordon, J. Ventura, L. Li, S. Thayumanavan, Multi-stimuli responsive macromolecules and their assemblies, Chem. Soc. Rev. 42 (2013) 7421-7435.

29 L.C. Yin, H.Y. Tang, K.H.N. Zheng, Z.Y. Song, N.P. Gabrielson, H. Lu, J.J. Cheng, Light-responsive helical polypeptides capable of reducing toxicity and unpacking DNA: toward nonviral gene delivery, Angew. Chem. Int. Ed. 52 (2013) 9182-9186.

30 W. Cao, Y. W. Gu, T. Y. Li, H.P. Xu, Ultra-sensitive ROS-responsive telluriumcontaining polymers, Chem. Commun. 51 (2015) 7069-7071.

31 R.C. Fang, H.P. Xu, W. Cao, L.L. Yang, X. Zhang, Reactive oxygen species (ROS)-responsive tellurium-containing hyperbranched polymer, Polym. Chem. 6 (2015) 2817-2821.

32 L. Wang, F. Q. Fan, W. Cao, H.P. Xu, Ultrasensitive ROS-pesponsive coassemblies of tellurium-containing molecules and phospholipids. ACS. Appl. Mater. Interfaces, 7 (2015) 16054-16060.

33 W. Cao, L.Wang, H.P. Xu, Selenium/tellurium containing polymer materials in nanobiotechnology, Nano Today10 (2015) 717-736.

34 L. Wang, W. Cao, H.P. Xu, Tellurium-containing polymers: towards biomaterials and optoelectronic materials, ChemNanoMat 2 (2016) 479-488. 
35 W. Cao, L. Wang, H. P. Xu, Coordination responsive tellurium-containing multilayer film for controlled delivery, Chem. Commun. 51 (2015) 5520-5522.

36 W. Cao, Y.W. Gu, M. Meineck, T.Y. Li, H.P. Xu, Tellurium-containing polymer micelles: competitive-ligand-regulated coordination responsive systems, J. Am. Chem. Soc. 136 (2014) 5132-5137.

37 Y.Y. Li, T. Wen, R.F. Zhao, X.X. Liu, T.J. Ji, H. Wang, X.W. Shi, J. Shi, J.Y. Wei, Y.L. Zhao, X.C. Wu, G.J. Nie, Localized electric field of plasmonic nanoplatform enhanced photodynamic tumor therapy, ACS Nano 8 (2014) 1152911542.

38 L. Viitala, S. Pajari, T. Lajunen, L.S. Kontturi, T. Laaksonen, P. Kuosmanen, T. Viitala, A. Urtti, L. Murtomaki, Photothermally triggered lipid bilayer phase transition and drug release from gold nanorod and indocyanine green encapsulated liposomes, Langmuir 32 (2016) 4554-4563.

39 M. Wang, S. Sun, C.I. Neufeld, B. Perez-Ramirez, Q.B. Xu, Reactive oxygen species-responsive protein modification and its intracellular delivery for targeted cancer therapy, Angew. Chem. Int. Ed. 53 (2014) 13444-13448.

40 S.S. He, Y.X. Qi, G.Z. Kuang, D.F. Zhou, J.Z. Li, Z. G. Xie, X.S. Chen, X.B Jing, Y.B. Huang, Single-stimulus dual-drug sensitive nanoplatform for enhanced photoactivated therapy, Biomacromolecules 17 (2016) 2120-2027. 
41 Q.H. Xu, C.L. He, C.S. Xiao, X.S. Chen, Reactive oxygen species (ROS) responsive polymers for biomedical applications, Macromol. Biosci. 16 (2016) 635-646.

42 M.L. Viger, M. Grossman, N. Fomina, A. Almutairi, Low power upconverted near-IR light for efficient polymeric nanoparticle degradation and cargo release, Adv. Mater. 25 (2013) 3733-3738.

43 R. Mo, T.Y. Jiang, W. J. Sun, Z. Gu, ATP-triggered anticancer drug delivery, Nat. Commun. 50 (2014) 67-74.

44 H. Wang, Y. Wu, R.F. Zhao, G.J. Nie, Engineering the assemblies of biomaterial nanocarriers for delivery of multiple theranostic agents with enhanced antitumor efficacy, Adv. Mater. 25 (2013) 1616-1622. 
Near-Infrared Light Stimuli-responsive Synergistic Therapy Nanoplatforms Based on the Coordination of Tellurium-containing Block Polymer and Cisplatin for Cancer Treatment Feng Li, Tianyu Li, Wei Cao, Lu Wang, and Huaping $\mathrm{Xu}^{*}$

Keywords: tellurium-containing polymer, cisplatin, drug-delivery, photo-responisve, coordination chemistry

Photo-responsive cisplatin delivery system: using the coordination chemistry between platinum and tellurium and the ultra-sensitive ROS responsiveness of tellurium, a CDDP delivery system with near-infrared light-responsive property is developed by encapsulating platinum and photosensitizer ICG in tellurium-containing polymer based nanoparticles simultaneously.

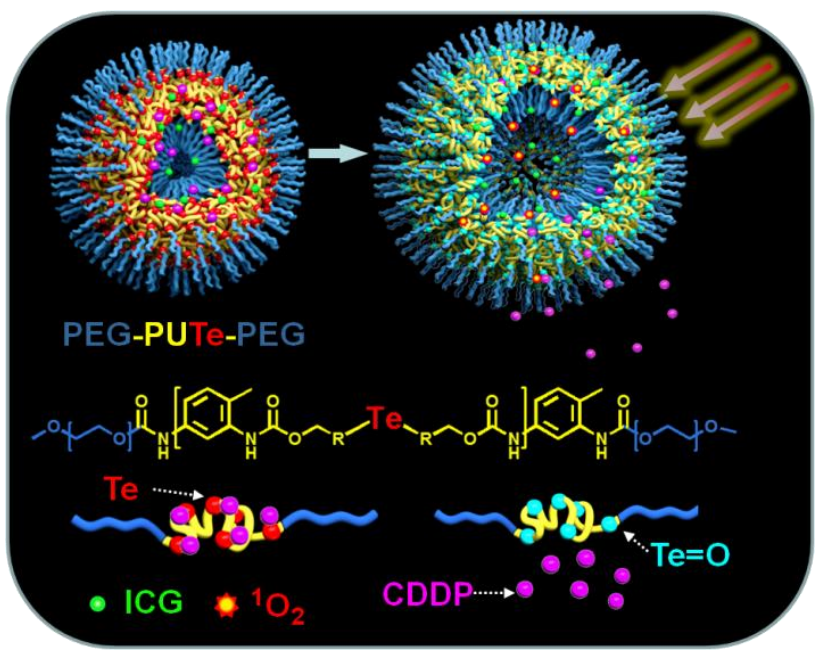

